\title{
Ma-Nuo-Xi decoction has an immunostimulating effect in cyclophosphamide-immunosuppressed mice
}

\author{
Duo Jie ${ }^{1,2 \#}$, Zhongshu Shan ${ }^{3 \#}$, Zhiliang $\mathrm{Ma}^{2}$, Ming Zhang ${ }^{1}$, Tingting Gao ${ }^{4}$, Hao $\mathrm{Wu}^{5}$, Bin Shan ${ }^{6}$, Lixin \\ Wei ${ }^{5}$, Hongtao $\mathrm{Bi}^{1 \wedge}$
}

${ }^{1}$ Qinghai Provincial Key Laboratory of Tibetan Medicine Pharmacology and Safety Evaluation, Northwest Institute of Plateau Biology, Chinese Academy of Science, Xining, China; ${ }^{2}$ State Key Laboratory of Tibetan New Drug Development, Institute of Tibetan Medicine of Qinghai Province, Xining, China; ${ }^{3}$ Department of Orthopaedics, People's Hospital of Qinghai Province, Xining, China; ${ }^{4}$ Department of Psychology, School of Public Health, Southern Medical University, Guangzhou, China; ${ }^{5}$ CAS Key Laboratory of Tibetan Medicine Research, Northwest Institute of Plateau Biology, Chinese Academy of Sciences, Xining, China; ${ }^{6}$ Qinghai Light Industry Institute Co. Ltd., Xining, China

Contributions: (I) Conception and design: D Jie, L Wei, H Bi; (II) Administrative support: H Wu, B Shan; (III) Provision of study materials or patients: Z Ma, Z Shan; (IV) Collection and assembly of data: M Zhang, T Gao; (V) Data analysis and interpretation: T Gao, H Bi; (VI) Manuscript writing: All authors; (VII) Final approval of manuscript: All authors.

\#These authors contributed equally to this work.

Correspondence to: Tingting Gao. Department of Psychology, School of Public Health, Southern Medical University, 1023 South Shatai Road, Guangzhou 510515, China. Email: gaott646@hotmail.com; Lixin Wei. CAS Key Laboratory of Tibetan Medicine Research, Northwest Institute of Plateau Biology, CAS, 23 Xin’ning Road, Xining 810008, China. Email: lxwei@nwipb.cas.cn; Hongtao Bi. Qinghai Provincial Key Laboratory of Tibetan Medicine Pharmacology and Safety Evaluation, Northwest Institute of Plateau Biology, CAS, 23 Xinning Road, Xining 810008, China. Email: bihongtao@hotmail.com.

Background: Ma-Nuo-Xi decoction (MNXD), as well as its hundreds of derivative preparations, has been used in Tibetan medicine since the 14th century. MNXD is in accordance with the theory of treatment determination based on syndrome differentiation. This study aimed to compare the effect of the auxiliary MNXD prescription (MNXD-AD) with that of the basic MNXD prescription (MNXD-BD) on the immunostimulating activity of MNXD.

Methods: The immunopotentiation of MNXD, MNXD-BD, and MNXD-AD was evaluated using a cyclophosphamide (CTX)-immunosuppressed mouse model. Their influences on non-specific and specific immunity were evaluated using immune organ indexes, peripheral white blood cell (WBC) count, red blood cell (RBC) count, platelet count, phagocytosis, macrophage-secreted nitric oxide (NO) and cytokines, natural killer (NK) cytotoxic activity, lymphocyte proliferation, serum cytokines, splenic T-lymphocyte subpopulations, and quantitative hemolysis of sheep red blood cell (QHS SRBC) assays.

Results: MNXD, MNXD-BD, and MNXD-AD increased the spleen and thymus indexes, as well as the peripheral WBC, RBC, and platelet counts. They also promoted phagocytosis, NO and cytokine secretion from macrophages, NK cytotoxic activity, and lymphocyte proliferation, and also raised the CD $4^{+} / \mathrm{CD} 8^{+}$ T-cell ratio, serum cytokine concentrations, and haemolysin formation in CTX-treated immunosuppressed mice. Compared with MNXD-BD and MNXD-AD, MNXD was superior in restoring the phagocytic index, concanavalin A (ConA)-induced T-lymphocyte proliferation, NO secretion from macrophages, and haemolysin formation, as well as the levels of interleukin 1 beta (IL-1ß), and serum interleukin-2 (IL-2) and interferon gamma (INF- $\gamma$ ).

Conclusions: MNXD, MNXD-BD, and MNXD-AD have excellent immunostimulating and myelosuppression-restoring activities on CTX-immunosuppressed mice. Among them, MNXD-AD might be an immunomodulator, which may happen to be in line with the clinical experience of Tibetan medicine physicians of using it to promote the efficacy of MNXD-BD.

$\wedge$ ORCID: 0000-0002-5569-8552. 
Keywords: Ma-Nuo-Xi decoction (MNXD); immunostimulating effect; cyclophosphamide-induced immunosuppression (CTX-induced immunosuppression); Tibetan medicine

Submitted Jun 09, 2020. Accepted for publication Sep 08, 2020.

doi: 10.21037/apm-20-1492

View this article at: http://dx.doi.org/10.21037/apm-20-1492

\section{Introduction}

Ma-Nuo-Xi decoction (MNXD), a Tibetan formulated medicine, comprises four medicinal materials: Tinospora sinensis (Lour.) Merr., Inula racemosa Hook.f., Rubus biflorus Buch., and Zingiber officinale Rosc., at a ratio of 20:20:6:5 $(\mathrm{w} / \mathrm{w})$ (1). Hundreds of Tibetan formulated medicines have been derived from this prescription since it was initially quoted in rGyud-bzhi between 748-765 $\mathrm{AD}$, earning it the name the "Mother of Decoction" $(2,3)$. Suka Ningni-duojie, who founded the South-school of Tibetan medicine in the 14th century, carried out thorough research of MNXD and its derivatives, and developed them to be the clinical drugs that are most in accord with the theory of treatment determination based on syndrome differentiation in Tibetan medicine (4). I. racemosa, T. sinensis, and R. biflorus comprised the basic prescription of MNXD, and Z. officinal was the auxiliary prescription that could be substituted with other medicinal materials according to the patient's specific symptoms (5). Currently, the recipes of MNXD and its derivative preparations are based on the experience of the treating clinician. The scientific basis for the addition of the auxiliary prescription to the basic prescription is still ambiguous.

T. sinensis, I. racemosa, R. biflorus, and Z. officinal. is the simplest recipe among the serial prescriptions of MNXD. According to the records in rGyud-bzhi (748-765 AD) and Somaradza (mid-13th century AD), the ability of MNXD to effectively relieve exterior syndrome and induce perspiration has led to its use as a treatment for plague, fever, rheumatoid arthritis, and other diseases (5). Hong et al. (3) found that the phagocytic index and ear tumidity of mice were significantly improved $(\mathrm{P}<0.05$ and $\mathrm{P}<0.01$, respectively) after 1 week of treatment with $0.5-2.0 \mathrm{~g} / \mathrm{kg}$ of MNXT granules. Chen et al. (6) found that the phagocytic index, ear tumidity, and the extent of hemolysis of sheep red blood cells (SRBC) were significantly promoted by the oral administration of $1.3-2.6 \mathrm{~g} / \mathrm{kg}$ of MNXT for 3 days in cyclophosphamide (CTX)-immunosuppressed mice $(\mathrm{P}<0.05)$. However, the effects of basic and auxiliary
MNXD prescriptions on immunomodulation have yet to be illuminated. To investigate the compatibility theory of MNXD, we aimed to compare the effect of the auxiliary MNXD prescription with that of the basic MNXD prescription on the immunostimulating effect of MNXD in CTX-immunosuppressed mice, by evaluating the immune organ index, peripheral white blood cell (WBC) count, red blood cell (RBC) count, platelet count, phagocytosis, macrophage-secreted nitric oxide (NO) and cytokines, natural killer (NK) cytotoxic activity, lymphocyte proliferation, splenic T-Lymphocyte subpopulations, serum cytokine, and quantitative hemolysis of SRBC (QHS) assays. We present the following article in accordance with the ARRIVE reporting checklist (available at http://dx.doi. org/10.21037/apm-20-1492).

\section{Methods}

\section{Plant materials and chemicals}

T. sinensis, I. racemosa, R. biflorus, and Z. officinale were purchased from Qinghai Fukang Pharmaceutical Company (Xining, China) on October 15, 2018, and identified by Prof. Yuzhi Du, Northwest Institute of Plateau Biology, Chinese Academy of Sciences (CAS) in Xining, China. The herbarium samples were recorded as IR20181015 for I. racemosa, TS20181015 for T. sinensis, RB20181015 for R. biflorus, and ZO20181015 for Z. officinale, and were deposited in Qinghai Provincial Key Laboratory of Tibetan Medicine Pharmacology and Safety Evaluation.

CTX was bought from Jiangsu Hengrui Medicine Co. (Lianyungang, Jiangsu, China). SRBCs, chicken red blood cells (CRBCs), guinea pig complements, RPMI1640 complete medium, and Hank's Balanced Salt Solution (HBSS) were purchased from Gibco, Thermo Fisher Scientific (Grand Island, NY, USA). Lipopolysaccharide (LPS), concanavalin A (ConA), 3-(4,5-dimethyl thiazolyl2)-2,5-diphenyl tetrazolium bromide (MTT), Giemsa stain, and limulus amebocyte lysate (LAL) assay kits were obtained from Sigma-Aldrich (St. Louis, MO, USA). 
Mouse interleukin (IL)-2, IL-4, and interferon (INF)- $\gamma$ enzyme-linked immunosorbent assay (ELISA) kits were purchased from Invitrogen, Thermo Fisher Scientific (Carlsbad, CA, USA). Tumor necrosis factor (TNF)- $\alpha$ and interleukin 1 beta (IL-1 $\beta$ ) ELISA kits, anti-CD4, and antiCD8 antibodies were bought from eBioscience of Thermo Fisher Scientific (San Diego, CA, USA). NO kits were obtained from Beyotime Biotechnology (Nantong, Jiangsu, China).

\section{Preparation of the herbal decoction}

MNXD preparation consists of four medicinal materials: $T$. sinensis, I. racemosa, $R$. biflorus, and Z. officinale (in a weight ratio of 20:20:6:5). MNXD-BD preparation is composed of three medicinal materials: T. sinensis, I. racemosa, and $R$. biflorus (in a weight ratio of 20:20:6). MNXD-AD preparation comprises Z. officinale alone. All three of the preparations were respectively decocted in boiling water at a solid-to-liquid ratio of 1:20 (w/v) for 3 hours. The the extracts were then centrifuged at 10,000 rpm for $30 \mathrm{~min}$ to remove insoluble residues, and the supernatants were concentrated to certain volumes to generate MNXD (full prescription decoction), MNXD-BD (basic prescription decoction), and MNXD-AD (auxiliary prescription decoction), which were stored at $-80{ }^{\circ} \mathrm{C}$ before use. The endotoxin content of the experimental samples was determined to be $<0.015 \mathrm{EU} / \mathrm{mg}$ using a LAL assay kit.

\section{Animals and treatment}

Six-to-eight-week-old male BALB/c mice were supplied by Charles River Laboratories (SCXK [Jing] 2016-0006; Beijing, China). The mice were housed under a 12/12-hour light-dark cycle at an ambient temperature of $25^{\circ} \mathrm{C}$. During the experiments, the mice were given free access to standard laboratory chow and distilled water.

Animal handling procedures were conducted according to the ARRIVE guidelines and the National Institutes of Health Guide for the Care and Use of Laboratory Animals (NIH Publications No. 8023). All animal experiments were approved by the ethics committee of Northwest Plateau Institute of Biology, CAS (NWIPB20171106-01).

After 1 week of acclimatization, the mice were randomly divided into 6 groups ( $\mathrm{n}=10$ in each group): the normal control group, the model control group, the positive control group (levamisole, an immunopotentiating agent), the
MNXD group, the MNXD-BD group, and the MNXD$\mathrm{AD}$ group. Apart from the normal control group, the mice were intraperitoneally injected with $50 \mathrm{mg} / \mathrm{kg}$ of CTX daily for 3 days, and then orally administered $0.2 \mathrm{~mL}$ of drugs for 14 days. The normal control group mice were only orally treated with $0.2 \mathrm{~mL}$ of sterile water per day for 14 days. In the positive control group, the dose of levamisole was $10 \mathrm{mg} / \mathrm{kg}$ bw/day. In the three experimental groups, the doses of MNXD, MNXD-BD, and MNXD-AD were $1.6,1.44$, and $0.16 \mathrm{~g} / \mathrm{kg}$ bw per day of the original plant materials, respectively, which are their clinically equivalent dosages $(1,7)$. Whole blood was collected 24 hours after the last administration from the orbit. After weighing, the mice were sacrificed by decapitation. Their spleens and thymuses were promptly collected, and the indices (the ratio of organ weight to body weight) were calculated.

\section{Peripheral WBC, RBC, and platelet counts}

The number of the peripheral WBCs, RBCs, and platelets were counted using an LH780 Hematology Analyzer (Beckman Coulter, Inc., Brea, CA, USA).

\section{Preparation of spleen cell suspension}

The spleens of the mice were minced in iced HBSS, and then pressed gently through a 200 -mesh metal sieve to remove major tissue aggregates. The filtrates were lysed with hemolysis solution $\left(7 \mathrm{~g} / \mathrm{L} \mathrm{NH}_{4} \mathrm{Cl}\right.$ and $2.6 \mathrm{~g} / \mathrm{L}$ Tris$\mathrm{HCl})$ and centrifuged at $180 \times \mathrm{g}$ for $10 \mathrm{~min}$ to remove the erythrocytes, and the spleen cell suspensions were finally obtained. The spleen cells were suspended in $1 \mathrm{~mL}$ of cell culture medium (RPMI-1640 complete medium containing $10 \% \mathrm{v} / \mathrm{v} \mathrm{FBS}, 100 \mathrm{U} / \mathrm{mL}$ of penicillin, and $0.1 \mathrm{mg} / \mathrm{mL}$ of streptomycin), and an MTT assay was performed to determine the cell viability (8).

\section{Phagocytic function of peritoneal macrophages}

After the last drug administration, the mice were immunized by intraperitoneal injection of $1 \mathrm{~mL}$ of $20 \%$ CRBCs, and then sacrificed 30 min later. Peritoneal cells were obtained by treating the peritoneal cavities of the mice with the peritoneal lavage. The phagocytic function of peritoneal macrophages was determined using a $4 \%$ Giemsa-PBS stain solution, and the phagocytic rate and phagocytic index were calculated as described by Gao et al. (8). 


\section{NK cytotoxic activity of splenocyte assays}

A splenocyte assay was carried out to detect NK cytotoxic activity as described by Tsa and Won (9). Briefly, $5 \times 10^{5}$ cells $/ \mathrm{mL}$ of splenocytes (effector cells, E) were mixed with YAC-1 cells (target cells, T) at an E:T ratio of 100:1. After incubation at $37^{\circ} \mathrm{C}$ in a humidified $5 \% \mathrm{CO}_{2}$ incubator for 20 hours, the mixture was centrifuged at $400 \times \mathrm{g}$ for 5 min, and then subjected to an MTT assay. NK cytotoxic activity was calculated as follows: NK cytotoxic activity (\%) $=\left(A_{\mathrm{T}}-\left(A_{\mathrm{S}}-A_{\mathrm{E}}\right)\right) / A_{\mathrm{T}} \times 100 \%$, where $A_{\mathrm{T}}$ is the absorbance value of the control target cells at $570 \mathrm{~nm}, A_{\mathrm{S}}$ is the absorbance value of the test samples at $570 \mathrm{~nm}$, and $A_{\mathrm{E}}$ is the absorbance value of the control effector cells at $570 \mathrm{~nm}$.

\section{Lymphocyte proliferation assay}

An aliquot of $100 \mu \mathrm{L}$ of the splenocyte suspension $\left(5 \times 10^{6}\right.$ cells $\left./ \mathrm{mL}\right)$ was seeded into each well of a 96 -well plate along with either $5 \mu \mathrm{g} / \mathrm{mL}$ ConA or $10 \mu \mathrm{g} / \mathrm{mL}$ LPS. After 48 hours of preincubation at $37{ }^{\circ} \mathrm{C}$ in a humidified $5 \% \mathrm{CO}_{2}$ incubator, the cell viability was determined by MTT assay (8).

\section{Splenic T-lymphocyte subpopulation assay}

For the splenic T-lymphocyte subpopulation assay, the splenocyte suspension was adjusted to a concentration of $1 \times 10^{6}$ cells $/ \mathrm{mL}$ and mixed with $10 \mu \mathrm{L}$ of either antiCD4 or anti-CD8 antibody. After 60 min of incubation at $4{ }^{\circ} \mathrm{C}$, the cells were washed twice with PBS, and then resuspended in $1 \%$ paraformaldehyde (PFA). The $\mathrm{CD}^{4+}$ and $\mathrm{CD}^{8+}$ T-lymphocyte counts were determined using a flow cytometer (BD FACSCalibur, NJ, USA) and expressed as the percentages of the total counts of $\mathrm{T}$ lymphocytes (10).

\section{Quantitative bemolysis of SRBC (QHS) assay}

On day 12 , mice were intraperitoneally injected with $0.2 \mathrm{~mL}$ of $10 \%$ SRBC suspension. After initial sensitization for 5 days, the splenocyte suspension was prepared and adjusted to a concentration of $1 \times 10^{6}$ cells $/ \mathrm{mL}$ in PBS, before incubation with $1 \mathrm{~mL}$ each of $0.2 \%$ SRBCs and $10 \%$ guinea pig serum complement at $37{ }^{\circ} \mathrm{C}$ for 1 hour. After centrifugation at 3,000 rpm for $3 \mathrm{~min}$, the supernatant was used to determine the extent of hemolysis of SRBCs at $413 \mathrm{~nm}(11)$.

\section{Determination of serum cytokines}

Blood samples were obtained from the mice by retro-orbital phlebotomy 24 hours after the last administration of drugs. Serum was obtained from the samples by centrifugation at 3,000 rpm for $3 \mathrm{~min}$. The IL-2, IL-4, and INF- $\gamma$ contents in the serum were assayed using ELISA kits.

\section{Preparation of peritoneal macrophages and determination of NO and cytokine secretion}

Peritoneal cells were obtained using a peritoneal lavage, and then incubated in a 6 -well plate at $37^{\circ} \mathrm{C}$ for 3 hours. After the removal of the non-adherent cells with cold PBS, the adhered peritoneal macrophages were collected. The macrophage suspension was seeded into 96-well plates at a concentration $4 \times 10^{5}$ cells/well. After 48 hours of culture, the supernatants were obtained, and the secretions of NO and cytokines were detected using ELISA kits.

\section{Statistical analysis}

To ensure a completely randomized experimental design, all data were presented as the mean \pm standard deviation (SD) and subjected to analysis of variance. Multiple $t$-tests were conducted to identify the differences among means using GraphPad Prism software (Version 7.00). $\mathrm{P}<0.05$ was considered to be statistically significant.

\section{Results}

\section{Effects of MNXD, MNXD-BD, and MNXD-AD on body weight and immune organ indexes}

CTX, which is currently the most widely used alkylation agent in tumor chemotherapy, often results in an immunosuppressive effect (12). In this study, the body weights and spleen and thymus indices of the model group were significantly decreased by intraperitoneal injection of CTX for 3 days; however, these decreases in CTX-treated mice were significantly ameliorated by oral administration of MNXD, MNXD-BD, or MNXD-AD for 14 days $(\mathrm{P}<0.05)$ (Table 1). The spleen and thymus indices in MNXD, MNXD-BD, and MNXD-AD groups were $39.32 \%$ and $65.91 \%, 33.71 \%$ and $94.31 \%$, and $37.45 \%$ and $67.05 \%$ higher, respectively, than those in the model group. However, no significant differences existed between 
Table 1 Effects of MNXD, MNXD-BD, and MNXD-AD on body weight, spleen and thymus indices, and peripheral WBC, RBC, and platelet counts in immunosuppressed mice

\begin{tabular}{|c|c|c|c|c|c|c|}
\hline Group & Body weight (g) & $\begin{array}{l}\text { Spleen indices } \\
(\mathrm{mg} / \mathrm{g})\end{array}$ & $\begin{array}{l}\text { Thymus indices } \\
\text { (mg/g) }\end{array}$ & WBC $\left(10^{9} / \mathrm{L}\right)$ & $\operatorname{RBC}\left(10^{12} / \mathrm{L}\right)$ & Platelets $\left(10^{11} / \mathrm{L}\right)$ \\
\hline Normal & $27.13 \pm 2.15$ & $3.39 \pm 0.31$ & $1.79 \pm 0.30$ & $7.89 \pm 0.92$ & $4.65 \pm 0.31$ & $5.8 \pm 0.61$ \\
\hline Levamisole $^{\Delta \Delta}$ & $23.55 \pm 1.82^{\mathrm{e}}$ & $3.63 \pm 0.61^{d}$ & $1.46 \pm 0.22^{e}$ & $7.16 \pm 0.72^{e}$ & $3.93 \pm 0.41^{\mathrm{e}}$ & $5.66 \pm 0.63^{\mathrm{e}}$ \\
\hline $\operatorname{MNXD}^{\Delta \Delta \Delta}$ & $26.07 \pm 1.89^{e}$ & $3.72 \pm 1.01^{\mathrm{c}}$ & $1.46 \pm 0.27^{\mathrm{e}}$ & $7.54 \pm 0.90^{\mathrm{e}}$ & $4.27 \pm 0.52^{\mathrm{e}}$ & $5.62 \pm 0.71^{e}$ \\
\hline MNXD-AD ${ }^{\Delta \Delta \Delta \Delta \Delta}$ & $24.78 \pm 1.91^{\mathrm{e}}$ & $3.67 \pm 0.33^{\mathrm{e}}$ & $1.47 \pm 0.32^{c}$ & $7.45 \pm 1.01^{\mathrm{e}}$ & $4.11 \pm 0.57^{e}$ & $5.53 \pm 0.52^{\mathrm{e}}$ \\
\hline
\end{tabular}

${ }^{\Delta}$, Model group: mice were treated with $50 \mathrm{mg} / \mathrm{kg}$ of CTX by intraperitoneal injection for 3 days and then orally administered $0.2 \mathrm{~mL}$ of sterile water for 14 days; ${ }^{\Delta \Delta}$, Levamisole group: mice were treated with $50 \mathrm{mg} / \mathrm{kg}$ of CTX by intraperitoneal injection for 3 days and then orally administered $10 \mathrm{mg} / \mathrm{kg}$ of levamisole for 14 days. ${ }^{\Delta \Delta \Delta}$, MNXD group: mice were treated with $50 \mathrm{mg} / \mathrm{kg}$ of CTX by intraperitoneal injection for 3 days and then orally administered $1.6 \mathrm{~g} / \mathrm{kg}$ of MNXD for 14 days; ${ }^{\Delta \Delta \Delta \Delta}$, MNXD-BD group: mice were treated with $50 \mathrm{mg} / \mathrm{kg}$ of CTX by intraperitoneal injection for 3 days and then orally administered $1.44 \mathrm{~g} / \mathrm{kg}$ of MNXD-BD for 14 days; ${ }^{\triangle \Delta \Delta \Delta \Delta}$, MNXD-AD group: mice were treated with $50 \mathrm{mg} / \mathrm{kg}$ of CTX by intraperitoneal injection for 3 days and then orally administered $0.16 \mathrm{~g} / \mathrm{kg}$ of MNXD-AD for 14 days. The values are presented as mean $\pm \mathrm{SD}, \mathrm{n}=10$. ${ }^{\mathrm{a}}, \mathrm{P}<0.01 ;{ }^{\mathrm{b}}, \mathrm{P}<0.001$ vs. the normal group; ${ }^{\mathrm{c}}, \mathrm{P}<0.05 ;{ }^{\mathrm{d}}, \mathrm{P}<0.01 ;{ }^{\mathrm{e}}, \mathrm{P}<0.001$ vs. the model group. MNXD, Ma-Nuo-Xi decoction; MNXD-BD, the basic MNXD prescription; MNXD-AD, the auxiliary MNXD prescription; WBC, white blood cell; RBC, red blood cell.

the three experimental groups. Moreover, the body weights of the mice in the MNXD, MNXD-BD, and MNXD$\mathrm{AD}$ groups, the spleen index in the MNXD group, and the thymus indexes in the MNXD-BD and MNXD-AD groups were all higher than those in the levamisole group, indicating a positive effect of immunostimulation induced by MNXD, MNXD-BD, and MNXD-AD on atrophy.

\section{Effects of MNXD, MNXD-BD, and MNXD-AD on bematopoietic function}

As shown in Table 1, the peripheral $\mathrm{RBC}, \mathrm{WBC}$, and platelet counts were significantly decreased in CTXimmunosuppressed mice, but were elevated remarkably by oral administration of MNXD, MNXD-BD, or MNXD-AD for 14 days. These results suggested that MNXD, MNXD$\mathrm{BD}$, and MNXD-AD can inhibit the myelosuppression induced by CTX. However, no significant differences existed between the three treatments.

\section{Effects of MNXD, MNXD-BD, and MNXD-AD on macrophages}

Macrophages play a pivotal role in the initiation and regulation of the immune response by interacting with lymphocytes and acting as phagocytic, microbiocidal, and tumoricidal effector cells (13). As shown in Figure 1A, the phagocytic rate and index of the CTX-treated model group were markedly lower than those of the normal group. Meanwhile, MNXD, MNXD-BD, and MNXD$\mathrm{AD}$ promoted the recovery of the phagocytic rate and index of peritoneal macrophages in CTX-immunosuppressed mice $(\mathrm{P}<0.001)$. Compared with the CTX-treated model group, the percent recoveries were $12.63 \%$ and $18.72 \%$ (MNXD), $16.67 \%$ and $11.10 \%$ (MNXD-BD), and $17.67 \%$ and $13.96 \%$ (MNXD-AD), respectively. In particular, the phagocytic index of the MNXD group was significantly higher than that of the MNXD-BD group $(\mathrm{P}<0.05)$.

The levels of cytokine and NO secretion by macrophages were also analyzed using the Griess method and ELISA. As shown in Figure 1B, CTX decreased the secretions of IL-1 $\beta$, TNF- $\alpha$, and NO from macrophages, but MNXD prevented this decrease $(\mathrm{P}<0.05)$. In the MNXD group, the secretions of IL- $1 \beta$, TNF- $\alpha$, and NO were increased by $49.60 \%, 14.62 \%$, and $83.97 \%$, respectively, compared with the model group. MNXD-BD and MNXD-AD also significantly promoted the secretion of IL- $1 \beta$ and $\mathrm{NO}$ in CTX-immunosuppressed mice $(\mathrm{P}<0.001)$; however, the increases in TNF- $\alpha$ secretion induced by these treatments were not significant. Furthermore, the NO level of the MNXD-AD group and the IL- $1 \beta$ and NO levels of the MNXD-BD group were significantly lower than those of 

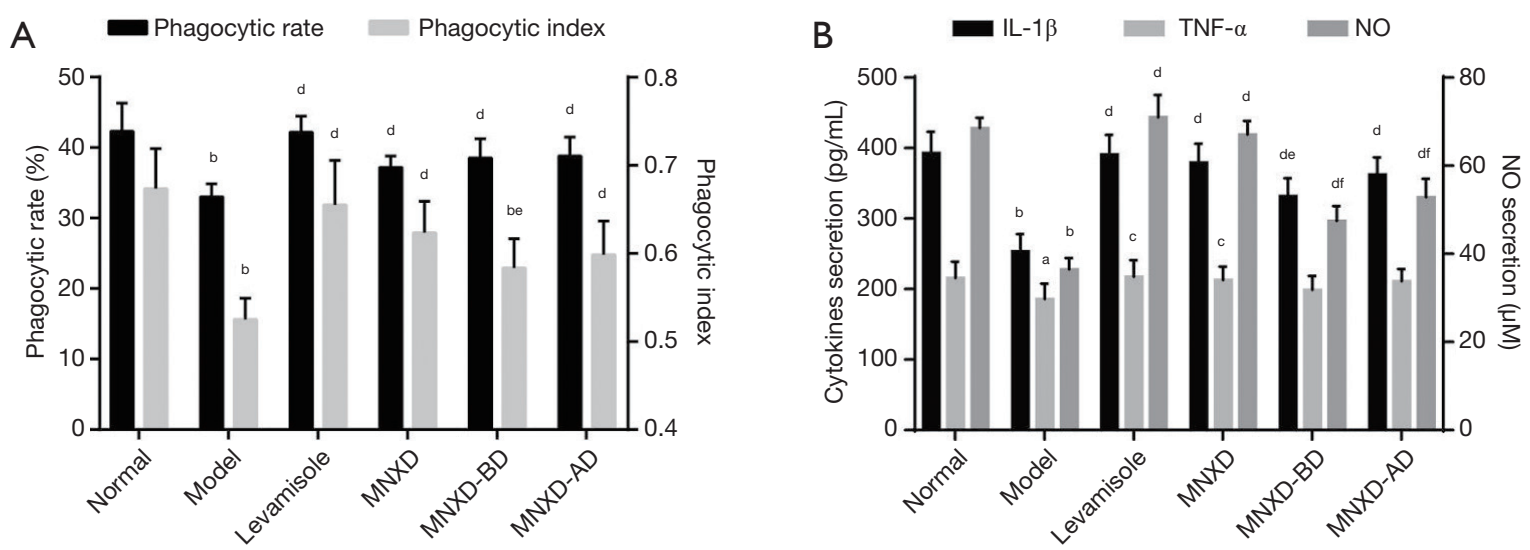

Figure 1 Effects of MNXD, MNXD-BD, and MNXD-AD on (A) phagocytosis, and (B) the secretion of IL-1 $\beta$, TNF- $\alpha$, and NO by macrophages in immunosuppressed mice. The values are presented as mean $\pm \mathrm{SD}, \mathrm{n}=10$. $^{\mathrm{a}}, \mathrm{P}<0.05 ;{ }^{\mathrm{b}}, \mathrm{P}<0.001$ vs. the normal group; ${ }^{\mathrm{c}}, \mathrm{P}<0.05$; ${ }^{\mathrm{d}}, \mathrm{P}<0.001$ vs. the model group; ${ }^{\mathrm{e}}, \mathrm{P}<0.05 ;{ }^{\mathrm{f}}, \mathrm{P}<0.001$ vs. the MNXD group. MNXD, Ma-Nuo-Xi decoction; MNXD-BD, the basic MNXD prescription; MNXD-AD, the auxiliary MNXD prescription; NO, nitric oxide.

Table 2 Effects of MNXD, MNXD-BD, and MNXD-AD on NK cytotoxic activity, CD4 $4^{+} \mathrm{CD} 8^{+}$ratio and serum haemolysin level in immunosuppressed mice

\begin{tabular}{|c|c|c|c|}
\hline Group & NK cytotoxic activity (\%) & $\mathrm{CD}^{+} / \mathrm{CD} 8^{+}$ratio & Serum haemolysin $\left(A_{413 n m}\right)$ \\
\hline Model $^{\Delta}$ & $20.61 \pm 2.91^{\mathrm{a}}$ & $2.14 \pm 0.32^{\mathrm{a}}$ & $0.33 \pm 0.03^{\mathrm{a}}$ \\
\hline Levamisole $^{\Delta \Delta}$ & $32.66 \pm 3.13^{d}$ & $3.22 \pm 0.27^{d}$ & $0.67 \pm 0.01^{d}$ \\
\hline$M N X D^{\Delta \Delta \Delta}$ & $23.97 \pm 3.64$ & $2.62 \pm 0.27^{\mathrm{b}}$ & $0.67 \pm 0.01^{d}$ \\
\hline MNXD-AD ${ }^{\Delta \Delta \Delta \Delta \Delta}$ & $28.47 \pm 3.49^{c}$ & $2.58 \pm 0.22^{b}$ & $0.59 \pm 0.04^{\mathrm{df}}$ \\
\hline
\end{tabular}

${ }^{\Delta}$, Model group: mice were treated with $50 \mathrm{mg} / \mathrm{kg}$ of CTX by intraperitoneal injection for 3 days, and then orally administered $0.2 \mathrm{~mL}$ of sterile water for 14 days; ${ }^{\Delta \Delta}$, Levamisole group: mice were treated with $50 \mathrm{mg} / \mathrm{kg}$ of CTX by intraperitoneal injection for 3 days, and then orally administered $10 \mathrm{mg} / \mathrm{kg}$ of levamisole for 14 days; ${ }^{\Delta \Delta \Delta}$, MNXD group: mice were treated with $50 \mathrm{mg} / \mathrm{kg}$ of CTX by intraperitoneal injection for 3 days, and then orally administered $1.6 \mathrm{~g} / \mathrm{kg}$ of MNXD of for 14 days; ${ }^{\Delta \Delta \Delta \Delta}$, MNXD-BD group: mice were treated with $50 \mathrm{mg} / \mathrm{kg}$ of CTX by intraperitoneal injection for 3 days, and then orally administered $1.44 \mathrm{~g} / \mathrm{kg}$ of MNXD-BD for 14 days; ${ }^{\Delta \Delta \Delta \Delta}$, MNXD-AD group: mice were treated with $50 \mathrm{mg} / \mathrm{kg}$ of CTX by intraperitoneal injection for 3 days, and then orally administered $0.16 \mathrm{~g} / \mathrm{kg}$ of MNXD-AD for 14 days. The values are presented as mean $\pm \mathrm{SD}, \mathrm{n}=10$. ${ }^{\mathrm{a}}, \mathrm{P}<0.001$ vs. the normal group; ${ }^{\mathrm{b}}, \mathrm{P}<0.05 ;{ }^{\mathrm{c}}, \mathrm{P}<0.01 ;{ }^{\mathrm{d}}, \mathrm{P}<0.001$ vs. the model group; ${ }^{\mathrm{e}}, \mathrm{P}<0.05 ;{ }^{f}, \mathrm{P}<0.001$ vs. the MNXD group. MNXD, Ma-Nuo-Xi decoction; MNXD-BD, the basic MNXD prescription; MNXD-AD, the auxiliary MNXD prescription; NK, natural killer; CTX, cyclophosphamide.

the MNXD group $(\mathrm{P}<0.05)$.

\section{Effects of MNXD, MNXD-BD, and MNXD-AD on NK cytotoxic activity}

NK cytotoxic activity is another essential function of the non-specific immune system (14). As shown in Table 2, only the cytotoxicity values of the MNXD-AD-treated group were significantly increased compared with the model group (38.14\%) $(\mathrm{P}<0.01)$. MNXD and MNXD-BD did not significantly abrogate the decrease in NK cytotoxic activity induced by CTX.

\section{Effects of MNXD, MNXD-BD, and MNXD-AD on splenic lymphocyte proliferation}

Lymphocytes are one of the essential components of the host defense against invading pathogens, and their 

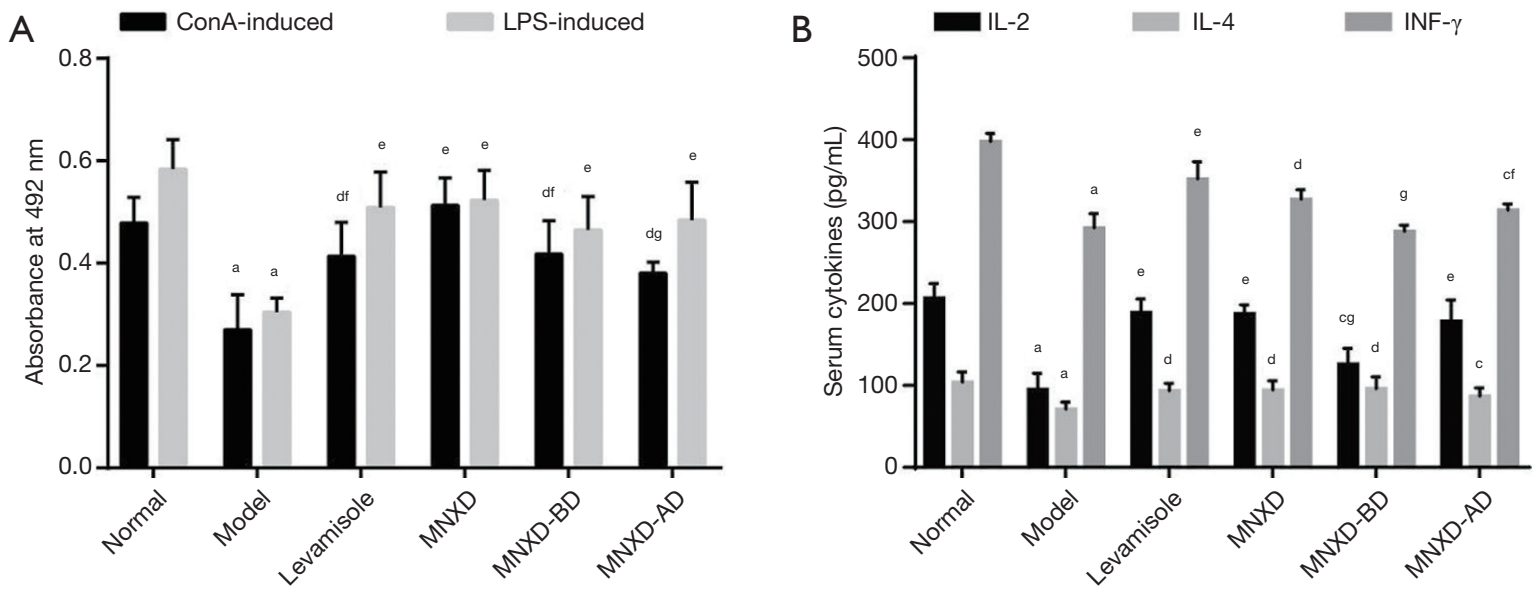

Figure 2 Effects of MNXD, MNXD-BD, and MNXD-AD on (A) splenic lymphocyte proliferation, and (B) cytokine concentrations in serum. The values are presented as mean $\pm \mathrm{SD}, \mathrm{n}=10$. ${ }^{\mathrm{a}}, \mathrm{P}<0.001 v s$. the normal group; ${ }^{\mathrm{c}}, \mathrm{P}<0.05 ;{ }^{\mathrm{d}}, \mathrm{P}<0.01 ;{ }^{\mathrm{e}}, \mathrm{P}<0.001$ vs. the model group; ${ }^{\mathrm{f}}, \mathrm{P}<0.05 ;{ }^{\mathrm{g}}, \mathrm{P}<0.001$ vs. the MNXD group. MNXD, Ma-Nuo-Xi decoction; MNXD-BD, the basic MNXD prescription; MNXD-AD, the auxiliary MNXD prescription.

proliferation is an important event in the activation cascade of both cellular and humoral immune responses (15). As shown in Figure $2 A$, treatment with MNXD, MNXD-BD, or MNXD-AD significantly enhanced the proliferations of ConA-induced T-cells and LPS-induced B-cells in CTX-immunosuppressed mice $(\mathrm{P}<0.01)$. Compared with the model group, the increases in the $\mathrm{T}$ - and $\mathrm{B}$-cell proliferation rates were $89.76 \%$ and $71.97 \%$ (MNXD), $54.71 \%$ and $52.71 \%$ (MNXD-BD), $40.96 \%$ and $59.15 \%$ (MNXD-AD), respectively. Moreover, MNXD showed significantly stronger abilities than levamisole, MNXD-BD, and MNXD-AD to augment T-lymphocyte proliferation $(\mathrm{P}<0.05)$.

\section{Effect of MNXD, MNXD-BD, and MNXD-AD on serum cytokine concentrations}

Cytokines are especially important in the immune system and modulate the balance between humoral and cell-based immune responses through receptors (16). As shown in Figure 2B, CTX significantly decreased the concentrations of IL-2, IL-4, and INF- $\gamma$ in the serum of mice $(\mathrm{P}<0.001)$. However, this effect was suppressed after the administration of MNXD, MNXD-BD, or MNXD-AD, as shown by the significant increases in the concentrations of these cytokines in the three groups (all $\mathrm{P}<0.05$, other than the concentration of INF- $\gamma$ in the MNXD-BD group). Furthermore, the concentrations of IL-2 and INF- $\gamma$ in the MNXD-BD group and the INF- $\gamma$ concentration in the MNXD-AD group were significantly lower than those in the MNXD group $(\mathrm{P}<0.05)$.

\section{Effect of MNXD, MNXD-BD, and MNXD-AD on splenic T-lymphocyte subpopulations}

T-lymphocyte subpopulations are closely associated with T-cell homeostasis and immune regulation. Among these subpopulations, $\mathrm{CD}^{+}{ }^{+} \mathrm{T}$-cells (helper T-lymphocytes, $\mathrm{Th}$ cells) and CD8 ${ }^{+}$T-cells (killer T-lymphocytes, Tc cells) are critical (17). As shown in Table 2 and Figure 3, the ratio of $\mathrm{CD}^{+} / \mathrm{CD}^{+}$was strikingly decreased in the model group compared to the normal group $(\mathrm{P}<0.001)$. In contrast, treatment with MNXD, MNXD-BD, and MNXD-AD significantly recovered the $\mathrm{CD} 4^{+} / \mathrm{CD} 8^{+}$ratio $(\mathrm{P}<0.05)$, inducing increases of $22.43 \%, 49.07 \%$, and $20.56 \%$, respectively, which suggested that oral administration of these decoctions can help to restore the ratio of splenic T-lymphocyte subpopulations.

\section{Effect of MNXD, MNXD-BD, and MNXD-AD on serum baemolysin formation}

To further investigate the effect of the three decoctions on the humoral immune response to cellular antigens, the serum levels of haemolysins were measured. As shown in Table 2, serum haemolysin production was significantly 

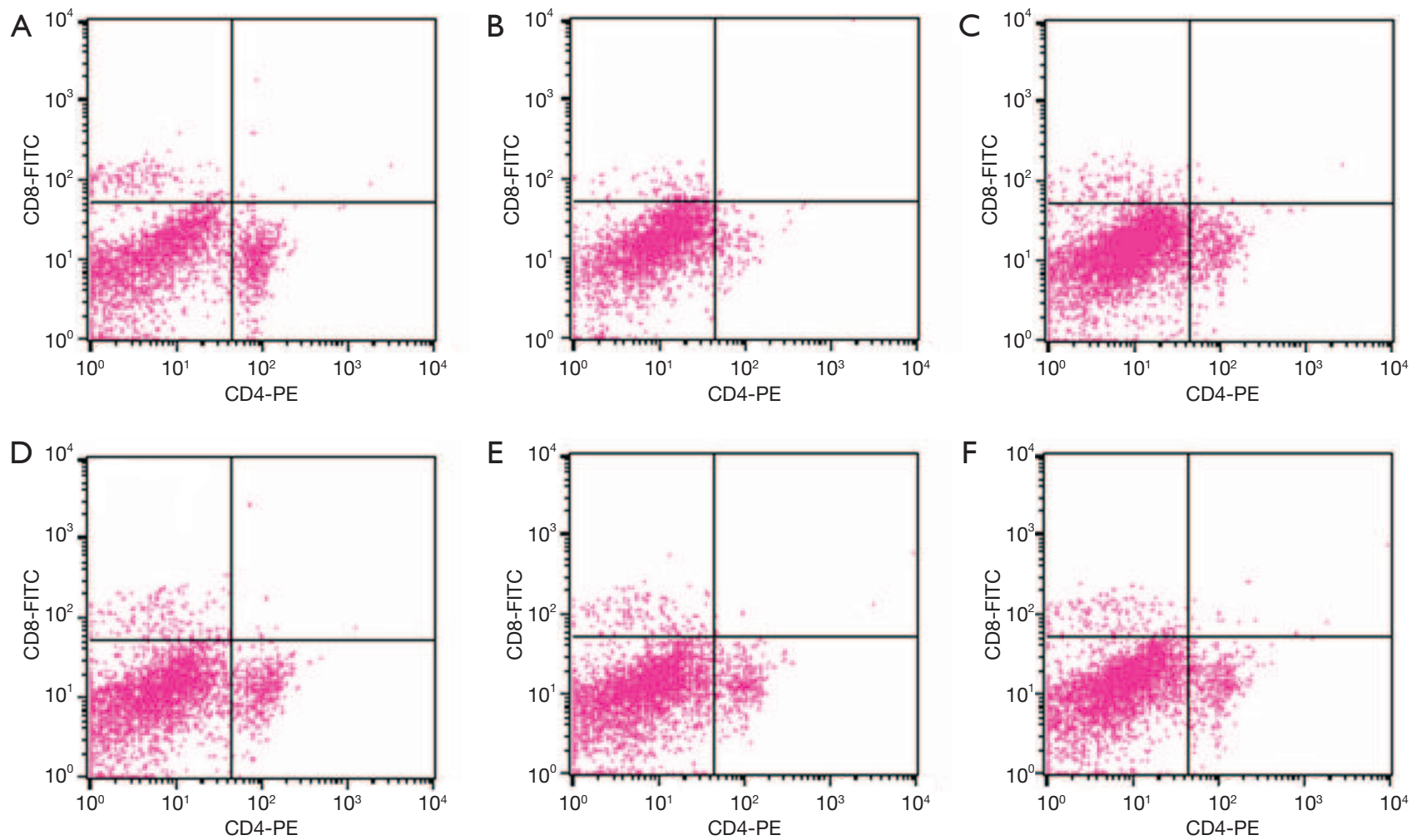

Figure 3 Effects of MNXD, MNXD-BD, and MNXD-AD on splenic T-lymphocyte subpopulations. (A) The normal group, (B) the model group, (C) the levamisole group, (D) the MNXD group, (E) the MNXD-BD group, and (F) the MNXD-AD group. MNXD, Ma-Nuo-Xi decoction; MNXD-BD, the basic MNXD prescription; MNXD-AD, the auxiliary MNXD prescription.

suppressed in the model group compared to the normal group $(\mathrm{P}<0.05)$. However, in the MNXD, MNXD-BD, and MNXD-AD groups, the serum haemolysin levels were significantly increased $(\mathrm{P}<0.001)$, with increases of $103.03 \%$, $96.97 \%$, and $78.79 \%$, respectively.

\section{Discussion}

In Tibetan medicine, MNXD and its derivative prescriptions are well known to be in line with the theory of treatment determination based on syndrome differentiation. However, to date, physicians still decide the composition of the herbs of these prescriptions without any experimental evidence, based only on personal experience and classical literature. In our published study, we aimed to investigate the immunostimulating effect of the polysaccharide components from Ma-Nuo-Xi decoction (18), but in the present study, we aimed to evaluate the immunopotentiation of MNXD, its basic prescription decoction (MNXD-BD), and its auxiliary prescription decoction (MNXD-AD), so as to elucidate the scientific basis for the contribution of auxiliary prescription to basic prescription. Since MNXD and its hundreds of derivative preparations, are the prescriptions that are well in line with the theory of treatment determination based on syndrome differentiation in Tibetan medicine, our findings will provide the scientific basis of the clinical usage of MNXD for Tibetan medicine physicians and promote the its modern development. In this study, we revealed that MNXD and its basic and auxiliary decoctions exerted immunomodulatory effects in CTX-induced immunosuppressed mice through regulating immune organs, immune cells, and immune cytokines. Treatment with MNXD, MNXD-BD, and MNXD-AD increased the spleen and thymus indexes and peripheral WBC count, and promoted phagocytosis, NO and cytokine secretion by macrophages, NK cytotoxic activity, and lymphocyte proliferation. Furthermore, there were also increases in the $\mathrm{CD} 4^{+} / \mathrm{CD}^{+} \mathrm{T}$-cell ratio, serum cytokine concentrations, and haemolysin formation. Moreover, peripheral RBC and platelet count assays showed that 
MNXD protected against CTX-induced myelosuppression.

In the prescription of MNXD, I. racemosa is considered as the "monarch drug", and its chemical profiling revealed the presence of alantolactones and isoalantolactones, sitosterol, daucosterol, inunolide, aplotexene, phenylacetonitrite, isoinunal, and aliphatic and eudesmalolide esters (19-22). Meanwhile, I. racemosa extracts were reported to have anti-atherogenic, cardioprotective, antiasthmatic, and antidiabetic activities; however, no immunostimulating activity has been noted to date (23-29). T. sinensis is another important component in the MNXD prescription. A variety of bioactive metabolites, such as tinosporinone, 1,1-dibenzoyl methane, allyloxyflavone, kokusaginine, menispermacide, palmatine, cycloeuphordenol, dinorditerpene glucosides, and lignan glucosides, have been isolated from T. sinensis (30). Pharmacological studies have indicated that the aqueous extract of $T$. sinensis led to remarkable increases in the WBC count of Swiss albino mice. Arabinogalactan from T. sinensis has been shown to be polyclonally mitogenic to $\beta$-cells and to augment antibody response as well as to enhance T-cell responses to model antigens, which suggests an immunomodulatory activity (31-33). In the basic prescription of MNXD, R. biflorus is the ingredient used in the lowest amount (by weight). It was revealed by chemical composition analysis to contain triterpenoids, steroids, and flavones, among which rutin and hyperoside were used as quality reference standards (34-36). To date, its pharmacological activity has not been reported. In this study, MNXD-BD, which is composed of the above three herbs, exhibited some immunomodulatory effects in CTX-induced immunosuppressed mice. However, the clinically equivalent dosage of MNXD-BD did not significantly promote macrophage secretion of TNF- $\alpha$ and $\mathrm{NO}$ or NK cytotoxic activity in CTX-treated mice.

As for the auxiliary prescription, Z. officinale (ginger) has been used as a raw material in many traditional preparations since ancient times. In Tibetan medicine, dried ginger is used for stomach ache, impotence, lung disease, vomiting, and diarrhea accompanied by cold extremities and a faint pulse (37). In recent years, the beneficial effects of ginger have been demonstrated in a vast range of clinical and experimental inflammatory disorders, and are considered to be mainly attributable to the gingerols, shogaols, zingerone, and paradols $(38,39)$. The immunological activity of ginger is consistent with the results of our study. The clinically equivalent dosage of MNXD-AD recovered non-specific and specific immunity in CTX-treated mice. Meanwhile, with the addition of MNXD-AD to MNXD-BD, more significant recoveries were observed in the phagocytic index, macrophage-secreted IL- $1 \beta$ and NO, ConA-induced T-lymphocyte proliferation, serum IL-2 and INF- $\gamma$ levels, and haemolysin formation, as compared with the addition of MNXD-BD. These results indicate that the addition of MNXD-AD to MNXD-BD produced a better effect on enhancing non-specific and specific immunity against CTXinduced immunosuppression. Thus, ginger is seemingly an immunomodulator, which may be in line with the clinical experience of Tibetan medicine physicians, who have used ginger to promote the efficacy of the basic prescription of MNXD. Some studies revealed that the possible therapeutic potentials of ginger in the treatment of the autoimmune diseases may exert mainly through the regulation of the Th1-, Th2-, Th9-, Th17-, Th22- and Treg cell-related immune responses, down-regulation of the B cell-related immune responses, modulation of the macrophagesrelated responses, modulation of the production of proand anti-inflammatory cytokines, down-regulation of the arachidonic acid-derived mediators, interfering with the toll like receptor-related signaling pathways, suppression of the inflammasomes, down-regulation of the oxidative stress, reduction of the adhesion molecules expression, and down-regulation of the expression of the chemokines and chemokine receptors (40-42). However, not all questions can be answered together in one study, so we will investigate the mechanism of immunostimulating effect of MNXD, MNXD-BD, and MNXD-AD in our further study, including detecting lysosomal enzyme and procoagulant activity of macrophage.

\section{Conclusions}

In this study, a CTX-immunosuppressed mouse model was established to evaluate the immunopotentiation of MNXD and its basic (MNXD-BD) and auxiliary (MNXD-AD) decoctions. The results showed that MNXD, MNXD$\mathrm{BD}$, and MNXD-AD significantly recovered the nonspecific and specific immunity of CTX-immunosuppressed mice, and exerted a protective effect against CTX-induced myelosuppression. Compared with MNXD-BD, MNXD obtained more significant recoveries in the phagocytic index, IL-1 $\beta$ and NO secretion by macrophages, ConAinduced T-lymphocyte proliferation, serum IL-2 and INF- $\gamma$ levels, and haemolysin formation, which indicates a better stimulating capacity produced by the addition 
of MNXD-AD to MNXD-BD against CTX-induced immunosuppression. Therefore, MNXD-AD (gingerdecoction) is potentially an immunomodulator, which may be in line with the clinical experience of Tibetan medicine physicians, who have used ginger to promote the efficacy of the basic prescription of MNXD.

\section{Acknowledgments}

Funding: This work was supported by National Key R\&D Program of China (2018YFC1708006), National Natural Science Foundation of China Regional Science Fund Project (81760396), China Postdoctoral Science Foundation-funded project (2019M652982), Innovation Platform Program (2017-ZJ-Y08) and Natural Science Foundation of Qinghai Province (2018-ZJ-902), Qinghai Provincial Health and Family Planning Medical and Health Science and Technology Project (2017-wjzdx-07), the International Partnership Program (153631KYSB20160004) and Central Asian Drug Discovery and Development Center of CAS (CAM201806), and Youth Innovation Promotion Association CAS.

\section{Footnote}

Reporting Checklist: The authors have completed the ARRIVE reporting checklist. Available at http://dx.doi. org/10.21037/apm-20-1492

Data Sharing Statement: Available at http://dx.doi. org/10.21037/apm-20-1492

Conflicts of Interest: All authors have completed the ICMJE uniform disclosure form (available at http://dx.doi. org/10.21037/apm-20-1492). The authors have no conflicts of interest to declare.

Ethical Statement: The authors are accountable for all aspects of the work in ensuring that questions related to the accuracy or integrity of any part of the work are appropriately investigated and resolved. Animal handling procedures were conducted according to the ARRIVE guidelines and the National Institutes of Health Guide for the Care and Use of Laboratory Animals (NIH Publications No. 8023). All animal experiments were approved by the ethics committee of Northwest Plateau Institute of Biology, CAS (NWIPB20171106-01).
Open Access Statement: This is an Open Access article distributed in accordance with the Creative Commons Attribution-NonCommercial-NoDerivs 4.0 International License (CC BY-NC-ND 4.0), which permits the noncommercial replication and distribution of the article with the strict proviso that no changes or edits are made and the original work is properly cited (including links to both the formal publication through the relevant DOI and the license). See: https://creativecommons.org/licenses/by-nc-nd/4.0/.

\section{References}

1. Chinese Pharmacopoeia Committee. Drug standards. Tibetan medicine by Ministry of Health PRC. Beijing: Pharmacopoeia Committee of Ministry of Health PRC, 1995.

2. Yundan-gongbao, Y. Gyud-bzhi. Lasa: Tibet People's Publishing Company, 1982.

3. Hong M, Liu Y, Han M, et al. Try to elucidate the pharmacodynamic effect of Ma-Nuo-Xi decoction. J Med Pharma Chin Minorities 2013;7:16-7.

4. Suka ND. Millions and Millions of Sarira of Tibetan Medicine. Lanzhou: Gansu Nationality Publishing Company, 1990.

5. He-Shang-Ma-Ha-Ya-Na, Bi-Ru-Zha-Na. Somaradza. Chengdu: Sichuan Nationality Publishing Company, 1990.

6. Chen X, Tong L, Wang J, et al. Effect of Si-wei-zang-muxiang powder on immune function of mice. Lishizhen Med Mate Med Res 2012;1:99-100.

7. Health Department of Qinghai Province. Tibetan medicine standards of Qinghai province. Xining: Health Department of Qingha Province, 1992.

8. Gao T, Bi H, Ma S, et al. The antitumor and immunostimulating activities of water soluble polysaccharides from Radix Aconiti, Radix Aconiti Lateralis and Radix Aconiti Kusnezoffii. Nat Prod Commun 2010;5:447-55.

9. Tsai YC, Won SJ. Effects of tramadol on T lymphocyte proliferation and natural killer cell activity in rats with sciatic constriction injury. Pain 2001;92:63-9.

10. Qi Z, Chen L, Li Z, et al. Immunomodulatory effects of (24R)-Pseudo-Ginsenoside HQ and (24S)-PseudoGinsenoside HQ on cyclophosphamide-induced immunosuppression and their anti-tumor effects study. Int J Mol Sci 2019;20:836.

11. Bin-Hafeez B, Haque R, Parvez S, et al. Immunomodulatory effects of fenugreek 
(Trigonella foenum graecum L.) extract in mice. Int Immunopharmacol 2003;3:257-65.

12. Manente FA, Quinello C, Ferreira LS, et al. Experimental sporotrichosis in a cyclophosphamideinduced immunosuppressed mice model. Med Mycol 2018;56:711-22.

13. Gordon $\mathrm{S}$. The role of the macrophage in immune regulation. Res Immunol 1998;149:685-8.

14. Barreira da Silva R, Münz C. Natural killer cell activation by dendritic cells: balancing inhibitory and activating signals. Cell Mol Life Sci 2011;68:3505-18.

15. Bi H, Gao T, Liu D, et al. Structures of $(1 \rightarrow 6)$-betaD-glucans from Bulgaria inquinans (Fries) and their immunological activities. Carbohydr Polym 2013;93:547-52.

16. Cabral GA, Ferreira GA, Jamerson MJ. Endocannabinoids and the immune system in health and disease. Handb Exp Pharmacol 2015;231:185-211.

17. Xing J, Luo K, Xiao Y, et al. Influence of CD4-1(+), CD4-2(+) and CD8(+) T lymphocytes subpopulations on the immune response of $\mathrm{B}$ lymphocytes in flounder (Paralichthys olivaceus) immunized with thymusdependent or thymus-independent antigen. Fish Shellfish Immunol 2019;84:979-86.

18. Jie D, Gao T, Shan Z, et al. Immunostimulating effect of polysaccharides isolated from Ma-Nuo-Xi decoction in cyclophosphamide-immunosuppressed mice. Int J Biol Macromol 2020;146;45-52.

19. Tan RX, Tang HQ, Hu J, et al. Lignans and sesquiterpene lactones from Artemisia sieversiana and Inula racemosa. Phytochemistry 1998;49:157-61.

20. Ma YY, Zhao DG, Gao K. Structural investigation and biological activity of sesquiterpene lactones from the traditional Chinese herb Inula racemosa. J Nat Prod 2013;76:564-70.

21. Zhang T, Gong T, Chen RY, et al. Two new trinoreudesmanolides from Inula racemosa. J Asian Nat Prod Res 2013;15:368-72.

22. Khan M, Mir SR, Ali A, et al. Aliphatic and eudesmalolide esters extracted from the roots of Inula racemosa Hook. Pharmacogn Mag 2014;10:40-6.

23. Lokhande PD, Dhaware BS, Jagdale SC, et al. Cardiac activity of isolated constituents of Inula racemosa. J Herb Pharmacother 2006;6:81-8.

24. Ojha S, Bharti S, Sharma AK, et al. Effect of Inula racemosa root extract on cardiac function and oxidative stress against isoproterenol-induced myocardial infarction. Indian J Biochem Biophys 2011;48:22-8.
25. Ojha S, Nandave M, Kumaria S, et al. Cardioprotection by Inula racemosa Hook in experimental model of myocardial ischemic reperfusion injury. Indian J Exp Biol 2010;48:918-24.

26. Pal HC, Sehar I, Bhushan S, et al. Activation of caspases and poly (ADP-ribose) polymerase cleavage to induce apoptosis in leukemia HL-60 cells by Inula racemosa. Toxicol In Vitro 2010;24:1599-609.

27. Arumugam P, Murugan M. Antimutagenic and Antiapoptotic Effects of Aqueous Root Extract of Inula racemosa Hook. f. on 4-NQO-Induced Genetic Damage in Mice. ISRN Pharmacol 2013;2013:768359.

28. Mohan S, Gupta D. Phytochemical analysis and differential in vitro cytotoxicity assessment of root extracts of Inula racemosa. Biomed Pharmacother 2017;89;781-95.

29. Kalachaveedu M, Raghavan D, Telapolu S, et al. Phytoestrogenic effect of Inula racemosa Hook $\mathrm{f}$ - A cardioprotective root drug in traditional medicine. J Ethnopharmacol 2018;210:408-16.

30. Haque MA, Jantan I, Abbas BS. Tinospora species: An overview of their modulating effects on the immune system. J Ethnopharmacol 2017;207:67-85.

31. Manjrekar PN, Jolly CI, Narayanan S. Comparative studies of the immunomodulatory activity of Tinospora cordifolia and Tinospora sinensis. Fitoterapia 2000;71:254-7.

32. Alamgir M, Uddin S. Recent Advances on The Ethnomedicinal Plants as Immunomodulatory Agents. Ethnomed Source Compl Therap 2010;2:227-44.

33. Narkhede AN, Jagtap SD, Kasote DM, et al. Comparative immunomodulation potential of Tinospora cordifolia (Willd.) Miers ex Hook. F., Tinospora sinensis (Lour.) Merrill and Tinospora cordifolia growing on Azadirachta indica A. Juss. Indian J Exp Biol 2014;52:808-13.

34. Kang SH, Shi YQ, Yang CX. Triterpenoids and steroids of root of Rubus biflorus. Zhong Yao Cai 2008;31:1669-71.

35. Kang SH, Zheng SZ. Two new flavones from Rubus biflorus Buch. Yao Xue Xue Bao 2007;42;1288-91.

36. Ma Z, Duo J. Study on Quality Standard of Tibetan Medicine Rubus biflorus. China Pharmacy 2018;2:179-82.

37. Dimaer D, Jizu M. The classics of Tibetan materia medica. Shanghai: Shanghai Scientific \& Technical Publishers, 2012.

38. Rahmani AH, Shabrmi FM, Aly SM. Active ingredients of ginger as potential candidates in the prevention and treatment of diseases via modulation of biological activities. Int J Physiol Pathophysiol Pharmacol 2014;6:125-36.

39. Prasad S, Tyagi AK. Ginger and its constituents: role 
in prevention and treatment of gastrointestinal cancer. Gastroenterol Res Pract 2015;2015:142979.

40. Jafarzadeh A, Nemati $M$. Therapeutic potentials of ginger for treatment of Multiple sclerosis: A review with emphasis on its immunomodulatory, anti-inflammatory and antioxidative properties. J Neuroimmunol 2018;324;54-75. 41. Tan BKH, Vanitha J. Immunomodulatory and

Cite this article as: Jie D, Shan Z, Ma Z, Zhang M, Gao T, Wu H, Shan B, Wei L, Bi H. Ma-Nuo-Xi decoction has an immunostimulating effect in cyclophosphamideimmunosuppressed mice. Ann Palliat Med 2020;9(5):3249-3260. doi: 10.21037/apm-20-1492
Antimicrobial Effects of Some Traditional Chinese Medicinal Herbs: A Review. Curr Med Chem 2004;11;1423-30.

42. Sharifi-Rad M, Varoni EM, Salehi B, et al. Plants of the genus Zingiber as a source of bioactive phytochemicals: from tradition to pharmacy. Molecules 2017;22;2145. 